\title{
THE LENGTH OF THE SHORTEST CLOSED GEODESIC IN A CLOSED RIEMANNIAN 3-MANIFOLD WITH NONNEGATIVE RICCI CURVATURE
}

\author{
EZEQUIEL BARBOSA AND YONG WEI
}

(Communicated by Michael Wolf)

\begin{abstract}
In this note we discuss the problem of finding an upper bound on the length of the shortest closed geodesic in a closed Riemannian 3-manifold in terms of the volume. More precisely, we show that there exists a positive universal constant $C$ such that, for every Riemannian 3-manifold $\left(M^{3}, g\right)$ with Ric $_{g} \geq 0$, at least one of the following assertions holds: (i). $\operatorname{Sys}_{g}(M) \leq$ $C \operatorname{Vol}_{g}(M)^{\frac{1}{3}}$, where $\operatorname{Sys}_{g}(M)$ denotes the length of the shortest closed geodesic in $M^{3}$; (ii). $M^{3}$ is diffeomorphic to $\mathbb{S}^{3}$ and there exists a closed minimal surface $\Sigma_{0}$ embedded in $M^{3}$, with index 1 , and $A_{g}\left(\Sigma_{0}\right) \leq C \operatorname{Vol}_{g}(M)^{\frac{2}{3}}$. This gives a partial answer to the problem proposed in Gromov's paper written in 1983.
\end{abstract}

\section{INTRODUCTION}

Given an $n$-dimensional closed Riemannian manifold $\left(M^{n}, g\right)$, it is an important problem to estimate the upper bound of the length of the shortest closed geodesic in $\left(M^{n}, g\right)$ in terms of the volume. In his seminal paper [6], Gromov proved that there exists an uniform constant $C_{1}(n)$ depending only on $n$ such that for every essential manifold $M^{n}$, we have

$$
\operatorname{Sys}_{1_{g}}(M) \leq C_{1}(n)\left(\operatorname{Vol}_{g}(M)\right)^{\frac{1}{n}},
$$

where $\operatorname{Sys}_{1 g}(M)$ is the least length of the noncontractible loop $\gamma$ in $M$ and the constant $C_{1}(n)$ can be taken as $6(n+1) n \sqrt[n]{(n+1) !}$. Here the manifold $M$ is said to be essential if there is an aspherical space $L$ and a map $f: M \rightarrow L$ such that $f$ represents a nonzero homology class in $H_{n}(L, G)$, with $G=\mathbb{Z}$ if $L$ is orientable and $G=\mathbb{Z}_{2}$ if $L$ is nonorientable.

Inequality (1.1) is often called the systolic inequality. Denote the length of the shortest nontrivial geodesic on $M$ by $\operatorname{Sys}_{g}(M)$. In any dimension, the shortest loop in every nontrivial homotopy class is a closed geodesic; then clearly we have $\operatorname{Sys}_{g}(M) \leq S y s_{1 g}(M)$. In general, there is the following question (see [6, p. 135] and [4, p. 115]).

Received by the editors November 7, 2014 and, in revised form, June 9, 2015 and October 30, 2015.

2010 Mathematics Subject Classification. Primary 53C42, 53C22.

Key words and phrases. Closed geodesic, systolic inequality, nonnegative Ricci curvature, minimal surfaces. 
Question 1.1. For an $n$-dimensional closed manifold $M^{n}$, is there a constant $C(M)$ such that for every Riemannian metric $g$ on $M$, we have

$$
\operatorname{Sys}_{g}(M) \leq C(M)\left(\operatorname{Vol}_{g}(M)\right)^{\frac{1}{n}} ?
$$

Moreover, the stronger question is whether the constant $C(M)=C(n)$ depends only on the dimension $n$.

This question is still open for many manifolds, e.g., for $M=\mathbb{S}^{n}, n \geq 3$. But it has a positive answer for all essential manifolds (see 6]) and for all closed surfaces (see [2, 3, 6, 8, 14]), with the universal constants $C(n)$ depending only on $n$. In particular, Loewner showed in 1949 (cf. 14]) that for every Riemannian metric on a torus $\mathbb{T}^{2}$

$$
\operatorname{Sys}_{1 g}\left(\mathbb{T}^{2}\right) \leq 2^{\frac{1}{2}} 3^{-\frac{1}{4}}\left(A_{g}\left(\mathbb{T}^{2}\right)\right)^{\frac{1}{2}},
$$

with equality holding if and only if it is a flat equilateral torus. Gromov [6] proved that every closed surface $\left(M^{2}, g\right)$ which is not a 2 -sphere satisfies

$$
\operatorname{Sys}_{1 g}(M) \leq \sqrt{\frac{\pi}{2}}\left(A_{g}(M)\right)^{\frac{1}{2}} ;
$$

while for the case of the 2-sphere, Croke 3 proved that every Riemannian metric $g$ on the 2-sphere $\mathbb{S}^{2}$ satisfies the estimate

$$
\operatorname{Sys}_{g}\left(\mathbb{S}^{2}\right) \leq 31\left(A_{g}\left(\mathbb{S}^{2}\right)\right)^{\frac{1}{2}} .
$$

The inequality (1.5) is not sharp and has been improved in [12], 15] and [16. In particular, the best known estimate was obtained by Rotman in [15],

$$
\text { Sys }_{g}\left(\mathbb{S}^{2}\right) \leq 4 \sqrt{2}\left(A_{g}\left(\mathbb{S}^{2}\right)\right)^{\frac{1}{2}}
$$

which is still not sharp, and it was suggested by Calabi and Croke that the sharp estimate should be $\operatorname{Sys} g\left(\mathbb{S}^{2}\right) \leq(12)^{\frac{1}{4}}\left(A_{g}\left(\mathbb{S}^{2}\right)\right)^{\frac{1}{2}}$. If one considers the convex hypersurfaces in $\mathbb{R}^{n+1}$, then Question 1.1 also has a positive answer which was shown independently by Treibergs [19] and Croke [3]. We refer the readers to the survey 4 for more information on this problem.

In a recent paper, Sabourau [17 considered a generalization of the above results about geodesics on closed surface in terms of minimal hypersurfaces and proved the following result.

Theorem 1.1 ([17]). Let $\left(M^{n}, g\right)$ be a closed Riemannian $n$-manifold with nonnegative Ricci curvature. There exists an embedded closed minimal hypersurface $\Sigma$ in $M$ with a singular set of Hausdorff dimension at most $n-8$ such that $A_{g}(\Sigma) \leq$ $C_{2}(n) \operatorname{Vol}_{g}(M)^{\frac{n-1}{n}}$, where $C_{2}(n)$ is an explicit positive constant depending only on $n$.

Inspired by Sabourau's theorem, in this paper we consider Question 1.1 for a three-dimensional closed manifold with nonnegative Ricci curvature. Our main result is the following.

Theorem 1.2. There exists a positive universal constant $C$ such that, for every closed Riemannian 3-manifold $\left(M^{3}, g\right)$ with Ric $_{g} \geq 0$, at least one of the following two assertions holds:

(1). $\operatorname{Sys}_{g}(M) \leq C \operatorname{Vol}_{g}(M)^{\frac{1}{3}}$, where $\operatorname{Sys} s_{g}(M)$ denotes the length of the shortest closed geodesic in $M^{3}$. 
(2). $M^{3}$ is diffeomorphic to $\mathbb{S}^{3}$ and there exists a closed minimal surface $\Sigma_{0}$ embedded in $M^{3}$, with index 1 , and $A_{g}\left(\Sigma_{0}\right) \leq C \operatorname{Vol}_{g}(M)^{\frac{2}{3}}$.

As a direct consequence, we obtain the following result.

Corollary 1.1. There exists a universal positive constant $C$ such that, for every closed Riemannian 3-manifold $\left(M^{3}, g\right)$ with Ric $c_{g} \geq 0$ and either (i). $\pi_{1}(M) \neq 0$; or (ii). $\pi_{1}(M)=0$ and there is no index 1 compact minimal surface in $\left(M^{3}, g\right)$, we have

$$
\operatorname{Sys}_{g}(M) \leq C \operatorname{Vol}_{g}(M)^{\frac{1}{3}},
$$

where $\operatorname{Sys}_{g}(M)$ denotes the length of the shortest closed geodesic in $M^{3}$.

Remark 1.1. There exist manifolds with nonnegative Ricci curvature and no closed minimal surface with index 1. For instance, there is no orientable closed minimal surface in $\mathbb{S}^{2} \times \mathbb{S}^{1}(r)$ with index 1 , when $r>1$. For more about this, see Theorem 4 in 20 .

Remark 1.2. After our paper was submitted awaiting a decision, a preprint by Liokumovich-Zhou 9] appeared on the arXiv. They proved that if a closed 3manifold $\left(M^{3}, g\right)$ has positive Ricci curvature and is not homeomorphic to a 3sphere, then $M$ contains a noncontractible closed geodesic of length at most $C \operatorname{Vol}_{g}(M)^{1 / 3}$, i.e.,

$$
\operatorname{Sys}_{1 g}(M) \leq C V \operatorname{Vl}_{g}(M)^{\frac{1}{3}} .
$$

The inequality (1.8) is slightly stronger than (1.7), as we usually have $\operatorname{Sys}_{g}(M) \leq$ $S y s_{1_{g}}(M)$. However, they need to impose a stronger assumption "Ric $c_{g}>0$ ". The proof of (1.8) in [9] is more involved by constructing a sweepout by 1-cycles of length at most $C \operatorname{Vol}_{g}(M)^{1 / 3}$.

\section{Proof of Theorem 1.2}

Proof. Given a closed Riemannian manifold $\left(M^{3}, g\right)$ with $R i c_{g} \geq 0$, from Hamilton's results [7] we obtain that the universal cover of $M^{3}$ is either $\mathbb{S}^{3}$ or $\mathbb{R}^{3}$ or $\mathbb{S}^{2} \times \mathbb{R}$. We consider the three cases separately.

If the universal cover of $M^{3}$ is $\mathbb{R}^{3}$, then $M^{3}$ is essential. Then, our theorem follows from Gromov's result (1.1),

$$
\operatorname{Sys}_{g}(M) \leq \operatorname{Sys}_{1_{g}}(M) \leq C_{1}(3) \operatorname{Vol}_{g}(M)^{\frac{1}{3}} .
$$

If the universal cover of $M^{3}$ is $\mathbb{S}^{2} \times \mathbb{R}$, then $M^{3}$ is either diffeomorphic to $\mathbb{P}^{3} \sharp \mathbb{P}^{3}$, $\mathbb{P}^{2} \times \mathbb{S}^{1}, \mathbb{S}^{2} \times \mathbb{S}^{1}$ or $\mathbb{S}^{2} \rtimes \mathbb{S}^{1}$. Here, $\mathbb{S}^{2} \rtimes \mathbb{S}^{1}$ denotes the nonorientable $\mathbb{S}^{2}$-bundle over $\mathbb{S}^{1}$, or equivalently the mapping torus of the antipodal map on $\mathbb{S}^{2}$. Since $\mathbb{P}^{3}$ and $\mathbb{P}^{2}$ are essential, we get that $\mathbb{P}^{3} \sharp \mathbb{P}^{3}$ and $\mathbb{P}^{2} \times \mathbb{S}^{1}$ are essential, respectively. In these cases, we again use Gromov's result. Hence, we consider the case where $M^{3}$ is diffeomorphic to $\mathbb{S}^{2} \times \mathbb{S}^{1}$. We can see that $\mathbb{S}^{2} \times \mathbb{S}^{1}$ is not essential. To justify this, note that the map $f: M \rightarrow L$ factors through the classifying map $M \rightarrow K\left(\pi_{1}(M), 1\right)$ and, for $M=\mathbb{S}^{2} \times \mathbb{S}^{1}, K\left(\pi_{1}(M), 1\right)=K(\mathbb{Z}, 1)=\mathbb{Z}$. Hence, $f_{*}[M]=0$ since $H^{3}\left(\mathbb{S}^{1} ; \mathbb{Z}\right)$ is trivial. Then, in this case, we cannot use Gromov's result. However, since the Ricci curvature is nonnegative, from the results in [11, §8], we can assume that $\left(M^{3}, g\right)$ is $\mathbb{S}^{2} \times \mathbb{S}^{1}$ with a product metric, i.e., the metric $g$ is a product metric on $\mathbb{S}^{2} \times \mathbb{S}^{1}$. The strategy here is to employ a kind of systole inequality for surfaces, proved by Loewner and Rotman. We consider the following two cases separately. 
If $\left(A_{g}\left(\mathbb{S}^{2}\right)\right)^{\frac{1}{2}} \leq \lambda L_{g}\left(\mathbb{S}^{1}\right)$, where $\lambda>0$ is a positive constant to be determined later and $A_{g}\left(\mathbb{S}^{2}\right), L_{g}\left(\mathbb{S}^{1}\right)$ denote the area of the surface $\mathbb{S}^{2}$ and length of $\mathbb{S}^{1}$ with respect to the induced metrics from $g$ respectively, let $\gamma$ denote the shortest closed geodesic in $\mathbb{S}^{2}$. By Rotman's result (1.6), we have

$$
L_{g}(\gamma) \leq 4 \sqrt{2}\left(A_{g}\left(\mathbb{S}^{2}\right)\right)^{\frac{1}{2}}
$$

where $L_{g}(\gamma)$ denotes the length of $\gamma$. Using $\left(A_{g}\left(\mathbb{S}^{2}\right)\right)^{\frac{1}{2}} \leq \lambda L_{g}\left(\mathbb{S}^{1}\right)$, we have

$$
\begin{aligned}
L_{g}(\gamma) & \leq 4 \sqrt{2}\left(A_{g}\left(\mathbb{S}^{2}\right)\right)^{\frac{1}{2}}=4 \sqrt{2}\left(A_{g}\left(\mathbb{S}^{2}\right)\right)^{\frac{1}{3}}\left(A_{g}\left(\mathbb{S}^{2}\right)\right)^{\frac{1}{6}} \\
& \leq 4 \sqrt{2} \lambda^{\frac{1}{3}}\left(A_{g}\left(\mathbb{S}^{2}\right)\right)^{\frac{1}{3}}\left(L_{g}\left(\mathbb{S}^{1}\right)\right)^{\frac{1}{3}} \\
& =4 \sqrt{2} \lambda^{\frac{1}{3}}\left(\operatorname{Vol}_{g}\left(\mathbb{S}^{2} \times \mathbb{S}^{2}\right)\right)^{\frac{1}{3}} .
\end{aligned}
$$

If $\left(A_{g}\left(\mathbb{S}^{2}\right)\right)^{\frac{1}{2}} \geq \lambda L_{g}\left(\mathbb{S}^{1}\right)$, we consider the torus $T^{2}=\gamma \times \mathbb{S}^{1}$ embedded in $\mathbb{S}^{2} \times \mathbb{S}^{1}$. From (1.3) we can find a closed geodesic $\beta$ in $T^{2}$ such that

$$
L_{g}(\beta) \leq 2^{\frac{1}{2}} 3^{-\frac{1}{4}}\left(A_{g}\left(T^{2}\right)\right)^{\frac{1}{2}},
$$

where $L_{g}(\beta)$ denotes the length of $\beta$ in $T^{2}$ with the induced metric. Note that the surface $T^{2}$ is totally geodesic in $\mathbb{S}^{2} \times \mathbb{S}^{1}$, so $\beta$ is also a geodesic in $\mathbb{S}^{2} \times \mathbb{S}^{1}$ and has the same length $L_{g}(\beta)$. Then,

$$
\begin{aligned}
L_{g}(\beta) & \leq 2^{\frac{1}{2}} 3^{-\frac{1}{4}}\left(A_{g}\left(T^{2}\right)\right)^{\frac{1}{2}}=2^{\frac{1}{2}} 3^{-\frac{1}{4}}\left(L_{g}(\gamma) L_{g}\left(\mathbb{S}^{1}\right)\right)^{\frac{1}{2}} \\
& \leq 2^{\frac{7}{4}} 3^{-\frac{1}{4}}\left(A_{g}\left(\mathbb{S}^{2}\right)\right)^{\frac{1}{4}}\left(L_{g}\left(\mathbb{S}^{1}\right)\right)^{\frac{1}{2}} \\
& =2^{\frac{7}{4}} 3^{-\frac{1}{4}}\left(A_{g}\left(\mathbb{S}^{2}\right)\right)^{\frac{1}{4}}\left(L_{g}\left(\mathbb{S}^{1}\right)\right)^{\frac{1}{3}}\left(L_{g}\left(\mathbb{S}^{1}\right)\right)^{\frac{1}{6}} \\
& \leq 2^{\frac{7}{4}} 3^{-\frac{1}{4}} \lambda^{-\frac{1}{6}}\left(A_{g}\left(\mathbb{S}^{2}\right)\right)^{\frac{1}{4}}\left(L_{g}\left(\mathbb{S}^{1}\right)\right)^{\frac{1}{3}}\left(A_{g}\left(\mathbb{S}^{2}\right)\right)^{\frac{1}{12}} \\
& =2^{\frac{7}{4}} 3^{-\frac{1}{4}} \lambda^{-\frac{1}{6}}\left(\operatorname{Vol}_{g}\left(\mathbb{S}^{2} \times \mathbb{S}^{1}\right)\right)^{\frac{1}{3}},
\end{aligned}
$$

where in the second inequality we used (2.1) and in the last inequality we used $L_{g}\left(\mathbb{S}^{1}\right) \leq \lambda^{-1}\left(A_{g}\left(\mathbb{S}^{2}\right)\right)^{\frac{1}{2}}$. Combining the above two cases, we obtain that if $M^{3}$ is diffeomorphic to $\mathbb{S}^{2} \times \mathbb{S}^{1}$, then there exists a geodesic $\gamma$ such that

$$
L_{g}(\gamma) \leq C\left(\operatorname{Vol}_{g}\left(\mathbb{S}^{2} \times \mathbb{S}^{1}\right)\right)^{\frac{1}{3}},
$$

where $C=\max \left\{4 \sqrt{2} \lambda^{\frac{1}{3}}, 2^{\frac{7}{4}} 3^{-\frac{1}{4}} \lambda^{-\frac{1}{6}}\right\}$. Clearly, we can choose $\lambda=2^{-\frac{3}{2}} 3^{-\frac{1}{2}}$ to obtain the optimal $C=2 / 3^{\frac{1}{6}}$. The nonorientable case $\mathbb{S}^{2} \rtimes \mathbb{S}^{1}$ now follows by passing to the oriented double covering $\mathbb{S}^{2} \times \mathbb{S}^{1}$.

Finally we assume that the universal cover of $M^{3}$ is $\mathbb{S}^{3}$. In this case, if $M^{3}$ is not simply connected, then, as it is well known, $M^{3}$ is essential and we can again use Gromov's inequality (1.1). Then we assume that $M^{3}=\mathbb{S}^{3}$. From Sabourau's result [17, there exists a closed embedded minimal surface $\Sigma$ in $\mathbb{S}^{3}$ such that

$$
A_{g}(\Sigma) \leq C_{2}(3)\left(\operatorname{Vol}_{g}\left(\mathbb{S}^{3}\right)\right)^{\frac{2}{3}} .
$$

Note that $\Sigma$ is orientable, since $M^{3}=\mathbb{S}^{3}$ and $\Sigma$ is an embedded closed minimal surface. Consider the set $\mathcal{E}$ of all closed minimal surfaces in $\mathbb{S}^{3}$. Let $\Sigma_{0}$ be the element in $\mathcal{E}$ with the least area. By Sabourau's inequality,

$$
A_{g}\left(\Sigma_{0}\right) \leq C_{2}(3)\left(\operatorname{Vol}_{g}\left(\mathbb{S}^{3}\right)\right)^{\frac{2}{3}} .
$$

If $\Sigma_{0}$ is stable, then from the second variation of the area functional, we have

$$
\int_{\Sigma_{0}}\left(R i c_{g}(\nu, \nu)+\left|A_{\Sigma_{0}}\right|^{2}\right) d \mu_{\Sigma_{0}} \leq 0
$$


where $\nu$ is a unit normal vector on $\Sigma_{0}$ and $\left|A_{\Sigma_{0}}\right|^{2}$ is the squared norm of the second fundamental form of $\Sigma_{0}$. Hence, from the inequality above and $R i c_{g} \geq 0, \Sigma_{0}$ is totally geodesic: $\left|A_{\Sigma_{0}}\right|^{2}=0$. Also, it follows from the proof of [18, Theorem 5.1] that $\Sigma_{0}$ is a sphere or a torus, since the scalar curvature $R_{g} \geq 0$ and $\Sigma_{0}$ is an orientable closed stable minimal surface. In fact, since $\Sigma_{0}$ is an orientable closed stable minimal surface in $M^{3}$, it follows from (2.4) and the Gauss equation that

$$
\begin{aligned}
0 \leq \int_{\Sigma_{0}} \frac{R_{g}}{2} d \mu_{\Sigma_{0}} & =\int_{\Sigma_{0}}\left(\operatorname{Ric}_{g}(\nu, \nu)+\frac{1}{2}\left|A_{\Sigma_{0}}\right|^{2}+K\right) d \mu_{\Sigma_{0}} \\
& \leq \int_{\Sigma_{0}}\left(\operatorname{Ric} g(\nu, \nu)+\left|A_{\Sigma_{0}}\right|^{2}\right) d \mu_{\Sigma_{0}}+2 \pi\left(1-g\left(\Sigma_{0}\right)\right) \\
& \leq 2 \pi\left(1-g\left(\Sigma_{0}\right)\right)
\end{aligned}
$$

where $K, g\left(\Sigma_{0}\right)$ are the Gauss curvature and genus of $\Sigma_{0}$ respectively. Then the genus of $\Sigma_{0}$ must be 0 or 1 , and therefore $\Sigma_{0}$ is a sphere or a torus. Applying Rotman's inequality (1.6) if $\Sigma_{0}$ is a sphere; or Loewner's inequality (1.3) if it is a torus, we obtain a closed geodesic $\gamma$ in $\mathbb{S}^{3}$ such that

$$
L_{g}(\gamma) \leq C \operatorname{Vol}_{g}\left(\mathbb{S}^{3}\right)^{\frac{1}{3}}
$$

where $C=\max \left\{4 \sqrt{2} \sqrt{C_{2}(3)}, 2^{\frac{1}{2}} 3^{-\frac{1}{4}} \sqrt{C_{2}(3)}\right\}$. If there exists $\Sigma$ in $\mathcal{E}$ such that $\Sigma \cap \Sigma_{0}=\emptyset$, then by [5. Lemma 3], we obtain again that $\Sigma_{0}$ is totally geodesic. Then we can again apply Rotman's inequality (1.6) and Gromov's inequality (1.1) to find a closed geodesic $\gamma$ in $\mathbb{S}^{3}$ such that

$$
L_{g}(\gamma) \leq C \operatorname{Vol}_{g}\left(\mathbb{S}^{3}\right)^{\frac{1}{3}}
$$

where $C=\max \left\{4 \sqrt{2} \sqrt{C_{2}(3)}, \sqrt{\frac{\pi}{2} C_{2}(3)}\right\}$. Now, assume that $\Sigma_{0}$ is not stable and every element $\Sigma$ in $\mathcal{E}$ satisfies $\Sigma \cap \Sigma_{0} \neq \emptyset$. Hence, as was done by Marques-Neves in [10, we obtain that $\Sigma_{0}$ has index 1 . For the convenience of the readers we present a proof of this. First, we get that $\mathbb{S}^{3} \backslash \Sigma_{0}$ is the disjoint union of two handlebodies: $N_{1}$ and $N_{2}$. Since $\Sigma_{0}$ is not stable, we can take a positive eigenfunction $\varphi \in C^{\infty}\left(\Sigma_{0}\right)$ for the lowest eigenvalue $\lambda<0$ of the Jacobi operator $L_{\Sigma_{0}}$, and consider a vector field $X$ on $\mathbb{S}^{3}$ such that $X=\varphi \nu$ on $\Sigma_{0}$, where $\nu$ is a unit normal vector pointing into $N_{1}$. Let $\left(F_{t}\right)_{t \in \mathbb{R}}$ denote the flow generated by the vector field $X$. Hence, we obtain

$$
\left.\frac{\partial}{\partial t}\left\langle H\left(\Sigma_{t}\right), \nu_{t}\right\rangle\right|_{t=0}=L_{\Sigma_{0}} \varphi=-\lambda \varphi>0
$$

Thus, there exists $\varepsilon>0$ small enough such that:

(i) $\Sigma_{t}=F_{t}\left(\Sigma_{0}\right)$ is contained in $N_{1}$ (in $\left.N_{2}\right)$ for all $0<t<\varepsilon($ for all $-\varepsilon<t<0)$;

(ii) the mean curvature vector $H\left(\Sigma_{t}\right)$ points into $N_{1}$ (into $N_{2}$ ) for all $0<t<\varepsilon$ (for all $-\varepsilon<t<0$ );

(iii) $A_{g}\left(\Sigma_{t}\right)<A_{g}\left(\Sigma_{0}\right)$ for all $0<|t|<\varepsilon$. 
Now, using Theorem 2.1 in 10 and the fact that every element in $\mathcal{E}$ intersects $\Sigma_{0}$, we can find a sweepout $\left(\Sigma_{t}\right)_{t \in[-1,1]}$ of $\mathbb{S}^{3}$ satisfying the following properties:

(a) $A_{g}\left(\Sigma_{t}\right)<A_{g}\left(\Sigma_{0}\right)$ for all $t \in[-1,1] \backslash\{0\}$;

(b) $\left(\Sigma_{t}\right)_{t \in[-1,1]}$ is smooth around $t=0$.

Consider then the function $f(t)=A_{g}\left(\Sigma_{t}\right)$. Hence,

$$
f^{\prime \prime}(0)=\int_{\Sigma_{0}}-\varphi L_{\Sigma_{0}} \varphi d \Sigma_{0}=\lambda \int_{\Sigma_{0}} \varphi^{2} d \Sigma_{0}<0 .
$$

To finish the proof we apply Proposition 3.1 in [10.

\section{Further REMARKS}

In this paper, we considered the closed 3-manifold with nonnegative Ricci curvature. For the general case, let $M^{3}$ be a closed 3-manifold. We say that $M^{3}$ is prime if a connected sum decomposition $M=P \sharp Q$ implies $P=\mathbb{S}^{3}$ or $Q=\mathbb{S}^{3}$, and it is said to be irreducible if every embedded 2-sphere bounds a 3-ball. A very wellknown result is that a closed prime 3-manifold is either an irreducible manifold, $\mathbb{S}^{2} \times \mathbb{S}^{1}$ or $\mathbb{S}^{2} \rtimes \mathbb{S}^{1}$. From the resolution of the Geometrization Conjecture given by G. Perelman, we have that a closed irreducible manifold is either an aspherical manifold, $\mathbb{P}^{2} \times \mathbb{S}^{1}$ or an elliptic space. Also, Gromov [6] observed that if a closed $n$-manifold $M$ is a connected sum $M=Q \sharp R$ and $Q$ is essential, then $M$ is also essential. Then, for the prime decomposition of a compact 3-manifold, every closed 3-manifold different from either $\mathbb{S}^{3}$, or $\sharp_{k}\left(\mathbb{S}^{2} \times \mathbb{S}^{1}\right)$ or $\sharp_{l}\left(\mathbb{S}^{2} \rtimes \mathbb{S}^{1}\right)$ is essential. Then, in this case, we can apply Gromov's result.

For the higher dimensional case, suppose $M^{n}(n \geq 4)$ is locally conformally flat with $R_{i c} \geq 0$. In this case, it follows from [13] that $M^{n}$ is diffeomorphic either to a quotient of $\mathbb{S}^{n}$, a quotient of $\mathbb{R}^{n}$ or a quotient of the cylinder $\mathbb{S}^{n-1} \times \mathbb{R}$. If $M^{n}$ is not simply connected and it is a quotient of the sphere $\mathbb{S}^{n}$, then $M^{n}$ is essential. Also, if $M^{n}$ is a quotient of the space $\mathbb{R}^{n}, M^{n}$ is essential. Again, we have to deal with the cases of the sphere $\mathbb{S}^{n}$ and the quotient of $\mathbb{S}^{n-1} \times \mathbb{R}$.

\section{ACKNOWLEDGEMENTS}

The first author wishes to express his gratitude to Professor André Neves for a number of enlightening conversations. Both authors would like to thank the referee for a very thorough reading and several useful suggestions, which improved the presentation of this paper. The first author was supported by FAPEMIG/Brazil and $\mathrm{CNPq} /$ Brazil grants, and the second author was supported by Jason D. Lotay throughout his EPSRC grant EP/K010980/1.

\section{REFERENCES}

[1] I. K. Babenko, Asymptotic invariants of smooth manifolds (Russian, with Russian summary), Izv. Ross. Akad. Nauk Ser. Mat. 56 (1992), no. 4, 707-751, DOI 10.1070/IM1993v041n01ABEH002181; English transl., Russian Acad. Sci. Izv. Math. 41 (1993), no. 1, 1-38. MR.1208148 (94d:53068)

[2] Yu. D. Burago and V. A. Zalgaller, Geometric inequalities, Grundlehren der Mathematischen Wissenschaften [Fundamental Principles of Mathematical Sciences], vol. 285, Springer-Verlag, Berlin, 1988. Translated from the Russian by A. B. Sosinskiı̈; Springer Series in Soviet Mathematics. MR936419 (89b:52020)

[3] Christopher B. Croke, Area and the length of the shortest closed geodesic, J. Differential Geom. 27 (1988), no. 1, 1-21. MR.918453(89a:53050) 
[4] Christopher B. Croke and Mikhail Katz, Universal volume bounds in Riemannian manifolds, Surveys in differential geometry, Vol. VIII (Boston, MA, 2002), Surv. Differ. Geom. vol. 8, Int. Press, Somerville, MA, 2003, pp. 109-137, DOI 10.4310/SDG.2003.v8.n1.a4. MR2039987 (2005d:53061)

[5] Gregory J. Galloway, A note on the fundamental group of a compact minimal hypersurface, Pacific J. Math. 126 (1987), no. 2, 243-251. MR869778(88a:53056)

[6] Mikhael Gromov, Filling Riemannian manifolds, J. Differential Geom. 18 (1983), no. 1, 1147. MR697984 (85h:53029)

[7] Richard S. Hamilton, Four-manifolds with positive curvature operator, J. Differential Geom. 24 (1986), no. 2, 153-179. MR862046 (87m:53055)

[8] James J. Hebda, Some lower bounds for the area of surfaces, Invent. Math. 65 (1981/82), no. 3, 485-490, DOI 10.1007/BF01396632. MR643566 (83e:53043)

[9] Y. Liokumovich and X. Zhou, Sweeping out 3-manifold of positive Ricci curvature by short 1-cycles via estimates of min-max surfaces, arXiv:1510.02896v2

[10] Fernando C. Marques and André Neves, Rigidity of min-max minimal spheres in threemanifolds, Duke Math. J. 161 (2012), no. 14, 2725-2752, DOI 10.1215/00127094-1813410. MR.2993139

[11] William Meeks III, Leon Simon, and Shing Tung Yau, Embedded minimal surfaces, exotic spheres, and manifolds with positive Ricci curvature, Ann. of Math. (2) 116 (1982), no. 3, 621-659, DOI 10.2307/2007026. MR678484 (84f:53053)

[12] A. Nabutovsky and R. Rotman, The length of the shortest closed geodesic on a 2-dimensional sphere, Int. Math. Res. Not. 23 (2002), 1211-1222, DOI 10.1155/S1073792802110038. MR.1903953(2003d:53062)

[13] Maria Helena Noronha, Some compact conformally flat manifolds with nonnegative scalar curvature, Geom. Dedicata 47 (1993), no. 3, 255-268, DOI 10.1007/BF01263660. MR.1235219 (94f:53068)

[14] P. M. Pu, Some inequalities in certain nonorientable Riemannian manifolds, Pacific J. Math. 2 (1952), 55-71. MR0048886 (14,87e)

[15] R. Rotman, The length of a shortest closed geodesic and the area of a 2-dimensional sphere, Proc. Amer. Math. Soc. 134 (2006), no. 10, 3041-3047 (electronic), DOI 10.1090/S0002-993906-08297-9. MR2231630 (2007f:53039)

[16] Stéphane Sabourau, Filling radius and short closed geodesics of the 2-sphere (English, with English and French summaries), Bull. Soc. Math. France 132 (2004), no. 1, 105-136. MR2075918 (2005g:53065)

[17] S. Sabourau, Volume of minimal hypersurfaces in manifolds with nonegative Ricci curvature, to appear in J. Reine. Angew. Math., DOI: 10.1515/crelle-2014-0147.

[18] R. Schoen and Shing Tung Yau, Existence of incompressible minimal surfaces and the topology of three-dimensional manifolds with nonnegative scalar curvature, Ann. of Math. (2) 110 (1979), no. 1, 127-142, DOI 10.2307/1971247. MR.541332 (81k:58029)

[19] Andrejs Treibergs, Estimates of volume by the length of shortest closed geodesics on a convex hypersurface, Invent. Math. 80 (1985), no. 3, 481-488, DOI 10.1007/BF01388728. MR791672 (86j:53069)

[20] Francisco Urbano, Second variation of one-sided complete minimal surfaces, Rev. Mat. Iberoam. 29 (2013), no. 2, 479-494, DOI 10.4171/RMI/727. MR3047425

Departamento de Matemática, Universidade Federal de Minas Gerais (UfmG), Caixa Postal 702, 30123-970, Belo Horizonte, MG, Brazil

E-mail address: ezequiel@mat.ufmg.br

Department of Mathematics, University College London, Gower Street, London, WC1E 6BT, United Kingdom

E-mail address: yong.wei@ucl.ac.uk 